\title{
High Tracheal Bifurcation as a Cause of Left Main Bronchus Obstruction in Infant: A case report from Saudi Arabia \\ Gawahir M. ${ }^{1}$, Khalid A.M. ${ }^{2}$, Safa E. ${ }^{3}$, Wadha A. ${ }^{4}$ \\ ${ }^{1}$ Pediatric Pulmonary division, King Fahad Medical City (KFMC), ${ }^{2}$ Pediatric Pulmonary Division, King Khalid Hospital/ King Saud University, ${ }^{3}$ Pediatric Pulmonary division, King Fahad Medical City(KFMC), \\ ${ }^{4}$ Pediatric Pulmonary and Sleep Physician, Head of the Pulmonary Unit, KFMC, KSA \\ Corresponding author: Gawahir M.A. Mukhtar, E-mail: jmukhtar@kfmc.med.sa, Mobile number: 00966543484521
}

\begin{abstract}
Background: Congenital short trachea (CST) an uncommon abnormality is defined as a reduction in the number of tracheal rings to 15 or less, compared to the average of 17 rings in normal infants. As a result, the carina is situated at a higher level than usual that lead the left main bronchus to course abnormally behind the arch of the aorta, making it prone to compression. CST is associated with a number of syndromes, including the Di George syndrome, several types of congenital heart disease, some skeletal dysplasias and meningomyelocoele. Case study: We report a case of one Saudi infant who presented with respiratory distress since birth and hyperinflation of left side of the lung secondary to extrinsic narrowing of proximal left main stem bronchus due to compression by arch of the aorta, and he underwent successful aortopexy of the aortic arch for this condition.
\end{abstract}

Keywords: High trachea bifurcation, children, Saudi Arabia, case report

\section{CASE REPORT}

An 8-week old infant was referred to us with history of respiratory distress since birth. He was a product of uneventful pregnancy, full term, normal spontaneous vaginal delivery (NSVD), birth weight was $3.2 \mathrm{~kg}$ and mother had good antenatal care. Immediately after birth developed respiratory distress and cyanosis and kept in NICU for 10 days, then transferred to other hospital where he had been admitted till age of 8 weeks, bronchoscopy was done for him and it was normal findings but he did not tolerate the procedure and developed immediately respiratory distress and transferred to pediatric intensive care unit (PICU), put on continuous positive airway pressure (CPAP) , weaned to oxygen via nasal cannula with persistent tachypnea and wheezes. Then transferred to our center for further management.

Serial chest radiographs showed progressive hyperinflation of the left lung suggestive of air trapping (Figure. 1)

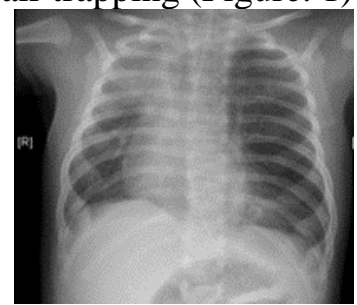

Figure (1): CXR showing Left side lung hyperinflation.

Computed tomography of the chest which was done in the previous hospital came with a report of three distinct areas of ground glass infiltrates in lower segment of the right upper lobe, the posterior segment of the right lower lobe and the posterior segment of the left lower lobe. A second opinion from our pediatric pulmonology and radiology teams confirmed the present of high tracheal bifurcation at a level of second thoracic vertebra which narrowing the proximal left main bronchus behind the aortic arch. (Figure $2 \& 3$ ).

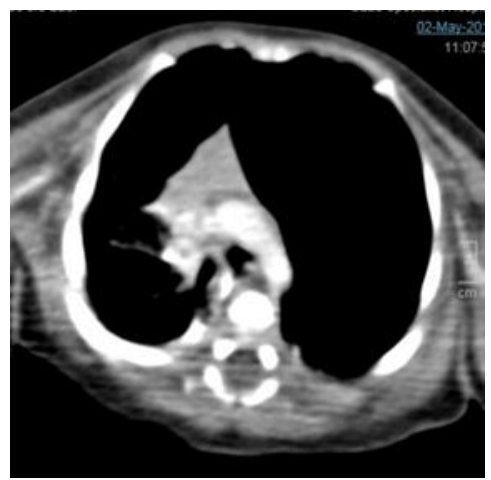

Figure (2): Axial sections mediastinal window showing the high bifurcation of the trachea in relation to arch of the aorta.

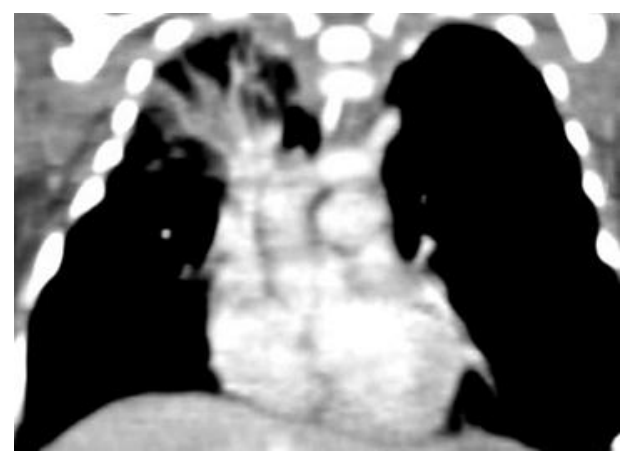

Figure (3): A coronal section mediastinal window showing the high bifurcation of the trachea in relation to arch of the aorta.

Echocardiography was done for him and it showed normal heart structures and functions.

Naso phayngolaryngeoscopy was done to rule out supraglottic stenosis, glottic abnormalities and it was normal. Barium swallow to rule out extrinsic esophageal compression was normal. 
Patient develop RSV bronchiolitis and became more distress, so shifted to PICU but did not required ventilation and discharged home after improvement with a plan for elective admission when he completely recover from the virus illness. The patient admitted electively after 3 weeks and a thoracoscopic aortopexy in right lateral position was done successfully and patient recovered smoothly without complications and discharged home after 10 days from the procedure in good condition without oxygen requirement.

In further follow up in outpatient clinic, patient clinically stable no more wheezes and gaining weight, chest radiography almost resolved of the hyperinflation. (Figure.4)

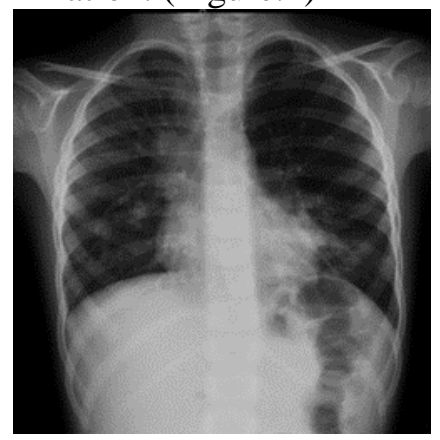

Figure (4): Chest radiography after thoracoscopic aortopexy.

\section{DISCUSSION}

Normal tracheal bifurcation is at the level of T4 vertebra at birth. The trachea grows in ring number and size until 2 years of age when it reached the T5 level ${ }^{(1)}$. CST defined as a decreased number of tracheal rings fewer than or equal to 15 and high bifurcation ${ }^{(1)}$. The major clinical problem has been related to advertent intubation (usually the right main bronchus) and subsequent complications like pneumothorax.

Despite it is an uncommon problem, it seems to be more frequent in certain conditions such as DiGeorge syndrome, skeletal dysplasias, brevicollis, congenital rubella syndrome, and interrupted aortic arch ${ }^{(2)}$. However, the long course of the left main bronchus renders it vulnerable to compression either behind the aortic arch or the ligamentum arteriosum ${ }^{(3)}$.

The true incidence of this abnormality has not been estimated. And the first report of a high tracheal bifurcation was published in 1979 by Hall ${ }^{(4)}$.

Sacco and his colleagues ${ }^{(5)}$ reported 4 girls who were evaluated for recurrent / chronic respiratory symptoms and found that a pulsatile extrinsic compression on the posterior bronchial wall of the left main stem, pressed between the descending aorta posteriorly and the left pulmonary artery anteriorly. The two arteries were closely linked together by a short length ligamentum that was resected with posterior aortopexy. Karthik ${ }^{(6)}$ reported a similar case of same age with same presentation of respiratory distress since birth and found same problem of high tracheal bifurcation at level of second thoracic vertebra and narrowing of the proximal left main bronchus behind the aorta and also he had successful aortopexy.

Plain radiograph can demonstrate the up normal high level of tracheal bifurcation, but still limited by reliance on patient position and exposure and angulation of the $\mathrm{x}$-ray beam ${ }^{(7)}$.

Computed tomography could show the high bifurcation of the trachea with more informative image with three dimensional reconstructions and consequently, we could receive higher information regarding the length of the trachea, angle of bifurcation, and the course and relationship of the bronchi to the surrounding structures ${ }^{(8)}$. The presence of bronchomalacia can be evaluated by direct flexible bronchoscopy or contrast bronchogram ${ }^{(8)}$. I n our case we avoided to repeat the bronchoscopy because the child did not tolerate the procedure before.

Successful management by aortopexy was done for this patient. The first description of aortopexy was on 1948 by Cross ${ }^{(9)}$ and it was for relief of obstruction of the trachea by innominate artery, the same principle can be used to remove the obstruction of the left main stem bronchus by aortic arch.

\section{CONCLUSION}

A high index of suspicion is needed to diagnose congenitally short trachea, since it is a rare cause of left main stem bronchus obstruction, and need to rule out other associated problems like bronchomalacia and congenital heart diseases. Aortopexy is the effective management to relief the obstruction. 


\section{REFERENCES}

1. Weil AL, Wells TR, Landing BH et al. (1989): Short trachea a hazard in tracheal intubation of neonates and infants: Syndromal associations, Anesthesiology, 71:367-373.

2. Wells TR, Well AL, Galvis DA, Senac MO, Landing BH, Vachon LA (1990): Diagnostic aspects and syndromal associatons of short trachea with bronchial intubation. Am J Dis Child, 144:1369-71.

3. Comerci SC, Berdon WE, Levin TL, RuzalShapiro C (1995): Congenitally short trachea and high carina with left mainstem bronchus compression by the ligamentum arteriosum. Pediatr Radiol., 25(1):194-6.

4. Hall RM (1979): High tracheal bifurcation, Anaesthesia, 34: 503-50

5. Sacco O, Santoro F, Ribera E et al. (2016): Short-length ligamentum arteriosum as a cause of congenital narrowing of the left main stem bronchus. Pediatr Pulmonol., 51(12):1356-1361.
6. Karthik R, Tom K, David K et al. (2014): High Tracheal Bifurication: An unusual cause of left Bronchial Obstruction. Ann Thorac Surg., 98:699-701.

7. Wells TR, Landing BH, Padua EM (1991): The question of parallax-effect on radiographic assessment of short trachea in infant and children. Pediatr Radiol., 21:490-3.

8. De priester JA, Vos GD, Van Wa ardenburg DA, Oei TK (1998): Congenitally Short Trachea with compression of the left main stem bronchus: MRI findings. Pediatr Radiol., 28:342-3.

9. Gross RE, Neuhauser EBD (1948): Compression of the trachea by anomalous innominate artery. An operation for its relief. Am J Dis Child., 75:570-4. 